\title{
Effects of Nurse Staffing on Surgical Patient-Outcomes with Cerebrovascular Disease
}

\author{
Sunyoung Jang \\ Department of Nursing, Hanseo University, Seosan, Korea \\ sjang@hanseo.ac.kr
}

\begin{abstract}
The purpose of this study was to examine the relationships between nurse staffing and patient outcomes including inpatient mortality and length of hospital stay.

Data were obtained from the '2008 Korea National Patients Survey' conducted by the Korea Institute for Health and Social Affairs. The study sample consisted of inpatients with surgical cerebrovascular diseases $(n=728)$. Nurse staffing was measured using 2 indicators: number of beds per nurse and number of inpatient days per nurse. Hospital characteristics included size, location, ownership, and physician staffing. Patient characteristics were age, gender, primary diagnosis, admission route, type of surgery, and primary payer. Patient outcomes were mortality during hospitalization and length of hospital stay. After controlling for hospital and patient characteristics, the relationships of nurse staffing to inpatient mortality and length of hospital stay were analyzed by multilevel analysis.

Nurse staffing was not significantly associated with inpatient mortality. Higher nurse staffing was related to a decrease in length of hospital stay.

The study findings suggested that higher nurse staffing might decrease length of hospital stay, which could increase efficiency and profits in providing hospital services. Government and nursing policies are required to ensure adequate nurse staffing in hospitals.
\end{abstract}

Keywords: Length of stay, Mortality, Multilevel, Patient outcomes, Nurse-staffing

\section{Introduction}

\subsection{Necessity of the Study}

Nurses are at the forefront of providing required patient services, since they are in the position to observe hospitalized patients more closely than anyone else in medical institutions [1]. The service provided by nurses have the capacity to significantly influence the patients not only physically but also psychologically, hence, the patients utilizing medical institution as well as the field of health care have taken interest in nurse staffing of each medical institution.

Studies have accordingly reported on the influence that the quality and the quantity of nursing depending on the level of nurse staffing has on patient outcomes [2-6]. These literature discuss the influence of nurse staffing on nursing sensitive patient outcomes that can be objectively measured including mortality, length of hospital stay, failure to rescue, cardiac arrest, shock, unexpected extubation, dyspnea, thrombosis, upper gastrointestinal bleeding, operation wound bleeding, fall, decubitus, nosocomial infection, urinary tract infection, hospital acquired pneumonia and nosocomial bloodstream infection [7]. Studies on the influence of nurse staffing on patient outcomes have been mostly conducted in the United States, however, the conclusions are inconsistent [8]. A large number of studies

\footnotetext{
${ }^{1}$ This manuscript is based on a part of the first author's doctoral dissertation from Hanyang University.
} 
have concluded that increased nurse staffing results in improved patient outcomes [12,4,9-10], while several have reported that nurse staffing does not significantly influence patient outcomes [3,11]. Such discrepancy may be the result of various factors influencing patient outcomes and the lack of definite control on the varied factors [12-13].

In Korea, self-developed questionnaires have been used to measure the level of influence of nurse staffing on patients [16]; alternatively, differential medical charge system for nurses has been implemented to monitor the change in nurse staffing in different hospitals [17], due to difficulties arising from deducing patient outcomes from objective data or confirmed factors that influence nurse staffing [14-15]. Park [18] examined the influence of the nurse staffing on objective patient outcomes, but their results cannot be generalized, as it included 82 general hospitals with more than 100 beds, out of the total of 254 general hospitals, in 2001.

Therefore, to further establish government policies for nurse staffing, research that controls the factors that influence patient outcomes through objective large-scale data is required. In Korea, large-scale data that includes such information on nurse staffing and patient outcomes is not yet well-established and thus, studies in nursing science have not progressed. Moreover, many factors that are influential to patient outcomes are neglected even in studies on large-scale national data. For such studies, it is critical to perform the analysis through consideration of revised hospital standards and patient standards [4].

Therefore, this study aimed to objectively examine the influence of nurse staffing on patient outcomes through multilevel analysis of large-scale national data and controlling factors that affect standards of patient outcome. The results $d$ from this study will provide evidence for the importance of the nation and medical institution's awareness on nurse staffing and adequate maintenance and management, and will contribute to the establishment of adequate government policies regarding nursing staff in the long term.

\subsection{Purpose of Study}

The purpose of the study was to analyze the relationship between nurse staffing and surgical cerebrovascular disease patient outcomes (mortality and length of hospital stay). The specific aims of the study were to:

1) Comprehend characteristics of hospitals and patients, and identify nurse staffing,

2) Analyze the relationship between nurse staffing and the mortality of surgical cerebrovascular disease patients,

and,

3) Analyze the relationship between nurse staffing and the length of hospital stay of surgical cerebrovascular disease patients.

\section{Methods}

This study was a descriptive correlation study, with multilevel analysis on the characteristics of hospital standards and relevant patient standards, in order to identify the relationship between nurse staffing and the mortality and length of hospital stay of surgical cerebrovascular disease patients.

\subsection{Data Source}

Patient survey is data collected through questionnaires developed by Korean Institute for Health and Social Affairs, distributed through health centers in cities and provinces, and recorded and submitted by the head or the employees at the medical institutions themselves (i.e. medical record administrator). Using the 2008 Patient Survey as the population, 26,362 medical institution listed in National Health Insurance Service's electronic data base on March 31, 2008, and complete enumeration survey was conducted 
in general hospitals, dental hospital, traditional herbal medicine clinic, public health service, health center, maternity center, and in clinic-level medical institution. Sample survey was on a sample size of 8,698; the number of surveyed institutions was 7,766; and the completion rate was at $89.3 \%$ [19]. Hospital and discharged patient data from among the medical institutions where complete enumeration survey was conducted were utilized in this study.

\subsection{Sample and Subjects}

In 2008, a total of 304 general hospitals were identified out of hospital categorization of the Patients Survey. Of these, 6 hospitals that had no nursing force (nurses and nurses' aide) were excluded; hence, 297 hospitals were included for the analysis. Among the total of 297 general hospitals, 286 that provided treatment for cerebrovascular disease (Korean Standard Classification of Diseases. I60-I69) patients, were selected for the analysis.

To obtain a statistically reliable estimation of the selected general hospitals, the hospitals with $<10$ surgery and non-surgery patients each were omitted according to the method of Cho et al on the Intensive Care Unit (ICU) patients [6]. The total number of general hospitals and patients included in the final study sample were as follows.

Table 1. Hospitals and Patients that were Included in the Final Study Sample

\begin{tabular}{|c|c|c|c|}
\hline Disease & $\begin{array}{c}\text { Korean Standard } \\
\text { Classification of Diseases } \\
\text { code }\end{array}$ & $\begin{array}{c}\text { Hospitals } \\
\text { number }\end{array}$ & $\begin{array}{l}\text { Patients } \\
\text { number }\end{array}$ \\
\hline subarachnoid hemorrhage & I60 & 40 & 194 \\
\hline intracerebral hemorrhage & I61 & 38 & 133 \\
\hline $\begin{array}{l}\text { other nontraumatic intracranial } \\
\text { hemorrhage }\end{array}$ & I62 & 28 & 71 \\
\hline cerebral infarction & I63 & 32 & 115 \\
\hline $\begin{array}{l}\text { occlusion and stenosis of } \\
\text { precerebral arteries, not resulting } \\
\text { in cerebral infarction }\end{array}$ & I65 & 13 & 36 \\
\hline other cerebrovascular disease & I67 & 36 & 175 \\
\hline $\begin{array}{l}\text { Sequelae of cerebrovascular } \\
\text { disease }\end{array}$ & I69 & 3 & 4 \\
\hline Total & & 41 & 728 \\
\hline
\end{tabular}

\subsection{Definition of Variables and their Measurement}

2.3.1. Nurse Staffing: The aim of this study was to determine the relationship between inpatient nurse staffing and patient outcomes. However, in the 2008 Korea National Patients Survey, nurses who care for both inpatients and outpatients were included. Thus, the calculation of the number of inpatient nurses was by a process of estimation. In this study, Medical Service Act was implemented to calculate the number of inpatient nurses.

For estimation of the number of inpatient nurses by the Medical Service Act, the Medical Service Act Enforcement Rule 38 (asterisk 5. Number of medical personnel at medical institutions) of Medical Service Act Item 36 Article 5 (number limitations of medical staff by types of medical institutions) was used as the fiducial point for general hospitals. 
Table 2. General Hospitals Nursing and Physician Staffing Standards Indicated by the Medical Service Act

\begin{tabular}{ll}
\hline \multicolumn{1}{c}{ Category } & \multicolumn{1}{c}{ General hospitals } \\
\hline Nurses & The annual average of inpatients per day divided by 2.5 (decimal points rounded up). \\
& 12 outpatients converted into 1 inpatient. \\
\hline
\end{tabular}

Hence, the number of inpatient nurses by Medical Service Act is calculated as follows:

-The number of nurse who care for inpatient=The number of total nurse $\times$ (The number of inpatient)/(The number of inpatient+(The number of outpatient/12)

- The number of beds per a nurse=The number of beds/The number of nurse who care for inpatients

-The number of inpatients per a nurse=The number of inpatients/ The number of nurse who care for inpatients

- Number of beds: The available beds data of the 2008 Korea National Patients Survey was utilized. Available beds is defined as actual beds in possession that can be made available irrelevant to government approval.

- Average number of inpatient days (hereafter referred to as 'the number of inpatients'): Refers to the number of inpatient days on the day of research in the 2008 Korea National Patients Survey.

\subsubsection{Hospital Characteristics}

- Location: The locations of hospitals were divided into the following regions for research: Seoul, Busan, Daegu, Incheon, Gwangju, Ulsan, Gyeonggi, Gangwon, Chungbuk, Jeonbuk, Jeonnam, Gyeongbuk, Gyeongnam and Jeju. However, in this study, we divided the hospitals by capital city (Seoul), metropolitan city (Busan, Daegu, Incheon, Gwangju, Daejeon, Ulsan) and province for analysis.

- Types of establishment: The medical institutions were categorized into public (national, special, public, others) and private (corporation, establish private).

- Existence of training center: There were no patient survey variables for training center, a variable with more than 1 specialist reported were classified as training center.

-Hospital Size: In the institution survey segment of the 2008 Korea National Patients Survey, the general hospitals that treat surgical patients were categorized into 100-499 beds and over 500 beds. An exception was made for hospitals that treat surgical inpatients as the number of subject hospitals was smaller, and they were categorized into 2 groups.

- Physician staffing level (number of beds per physician): Physician staffing level was defined as the number of beds per physician. The total number of physicians was calculated as the sum of specialists, general practitioners and medical residents of the 2008 Korea National Patients Survey, and the number of inpatients per physician was estimated through Medical Service Act as standard (3 outpatients being equivalent to 1 inpatient).

-The number of beds per a physician=Total number of beds $\div$ Total number of physician $\times$ (The number of inpatient)/(The number of inpatient+(The number of outpatient/3)) 


\subsubsection{Patient Characteristics}

- Discharged Patients' Residence: In this study, the discharged patients' residence were categorized into capital city (Seoul), metropolitan city (Busan, Daegu, Incheon, Gwangju, Daejeon, Ulsan), cities in terms of administrative districts and provinces for analysis.

- Major diagnosis: Refers to the main cause of the hospital visit i.e., disease or other reasons (vaccination, check-up etc.), and disease categorization refers to categorization according to "Korean Standard Classification of Diseases (KCD-5, 2007)". In this study, 'major disease classification number' variable from discharged patient survey of the 2008 Korea National Patients Survey was utilized.

- Major Surgery: Refers to the name of the main surgery performed on the patient during their visit to the medical institution; and surgery classification number refers to categorization according to "ICD-9-CM". For the purposes of this study, "major surgery classification number' variable from the discharge patients survey of 2008 Korea National Patients Survey was utilized.

- Admission route: Refers to the route via which a patient was hospitalized: admission as outpatient, admission through the emergency room or admission through alternate routes.

- Route of hospital visit: Refers to the route that the patients have come to the hospital, and were categorized into direct visit (coming to the hospital/medical institution without medical request from other medical institutions) and request from other institutions (request for treatment from other medical institutions, only the cases where the written request was attached).

- Primary payer: Refers to the means through which a patient paid for his or her medical fees, and were categorized into full sum by one's own expense (paying for the medical fees in full with their own money), health insurance (paying for the medical fee through health insurance, by National Health Insurance Act), medical benefit (those deemed eligible for medical benefit by the Medical Care Assistance Act) and occupational health and safety insurance - car insurance (the medical fee taken care of by the occupational health and safety insurance or the car insurance).

\subsubsection{Patient outcomes}

- Mortality: Refers to the cases that were marked as dead in 'patient outcomes' among the patient survey variables.

- Length of hospital stay: Refers to the number of days from day of hospitalization to day of leaving the hospital, and uses the 'length of hospital stay' variable in the patient survey.

\subsection{Analysis Method}

SAS Ver.9.2 program was used for the data analysis in this study, and the data was compiled into statistics through multilevel regression model and descriptive statistics in order to reflect the data as assemblage data of the patients in the hospital. The level of significance was set as $\mathrm{p}<.05$. The detailed methods of analysis were as follows.

1) In order to identify the general characteristics of subject medical institution, the characteristics of nursing outcome and nurse staffing, descriptive statistics such as mode, percentage, mean and standard deviation were used for the analysis.

2) To examine the relationship between nurse staffing and patient outcomes, linear regression and logistical regression multilevel analysis (PROC GLIMMIX) was performed. 
3) Logistical multilevel regression analysis was applied to patient mortality. As length of hospital stay was not within normal distribution, $\log$ (natural $\log$ ) was used in a linear multilevel regression model.

4) The correlation between the 2 indicators of nurse staffing (the number of beds per nurse and the number of inpatient days per nurse) was high, thus they were analyzed as 2 separate models in multilevel analysis. Model 1 included the number of beds per nurse indicator and Model 2 included the number of inpatient days per nurse indicator.

\subsection{Ethical Considerations}

This data utilized the original data of the 2008 Korea National Patients Survey. A request for the original data of the 2008 Korea National Patients Survey was submitted to Korean Institute for Health and Social Affairs through the internet. Documents requested by the institutes such as the research plan and a written oath were prepared subsequently and the original data was provided after internal evaluation. Prior to the commencement of the analysis of the provided original data, the study received approval (HYI-11-016) from the IRB of $\mathrm{H}$ general hospitals.

\section{Results}

\subsection{General Characteristics of Hospitals}

After examining the frequency according to the characteristics of the 41 hospitals in which surgical cerebrovascular disease patients were hospitalized, there were a total of 4 hospitals with $100-499$ beds $(9.8 \%)$ and 37 hospitals with over 500 beds $(90.2 \%)$. There were 35 corporate hospitals $(80.5 \%)$ and 39 training center $(95.1 \%)$, comprising the majority. After dividing the location of hospitals into capital city, metropolitan city, provinces, the hospitals based in the metropolitan city took up the majority with 15 $(36.6 \%)$ followed by 14 hospitals in provinces $(34.2 \%)$ and 12 in metropolitan cities (29.3\%), and all hospitals operated ICU.

Table 3. Hospital Characteristics

\begin{tabular}{lc}
\hline \multicolumn{1}{c}{ Categories } & The number of hospitals $(\%)$ \\
\hline The number of bed & \\
$100-499$ bed & $4(9.8)$ \\
over 500 bed & $37(90.2)$ \\
Type of establish & \\
Public & $9(19.5)$ \\
Private & $33(80.5)$ \\
Training physician or not & \\
Training hospital & $39(95.1)$ \\
No- Training hospital & $2(4.9)$ \\
Location of hospitals & \\
Special city & $15(36.6)$ \\
Metropolitan cities & $12(29.3)$ \\
Etc. & $14(34.2)$ \\
Having intensive care units & $41(100.0)$ \\
\hline Total & $41(100.0)$ \\
\hline
\end{tabular}

\subsection{Nurse Staffing}

Nurse staffing for 728 surgical cerebrovascular disease patients at 41 hospitals was examined. The estimated number of beds per nurse, Medical Service Act standard 
estimation was an average $1.9 \pm 0.4$ beds and the average number of inpatient days per nurse was $1.6 \pm 0.3$.

\subsection{General Characteristics of Patients}

The general characteristics of the 728 surgical cerebrovascular disease patients showed an average age of 56.8 years, with 377 male patients (51.8\%). Categorized per their diseases, subarachnoid hemorrhage (I60) patients were 194 (26.7\%), intracerebral hemorrhage (I61) patients were 133 (18.3\%), other nontraumatic intracranial hemorrhage (I62) patients were 71 (9.8\%), cerebral infarction (I63) patients were 115 (15.8\%), occlusion and stenosis of precerebral arteries, not resulting in cerebral infarction (I65) patients were 36(5.0\%), other cerebrovascular disease (I67) patients were 175 (24.0\%) and aftereffects of cerebrovascular disease (I69) patients were $4(0.6 \%)$, showing that the subarachnoid hemorrhage patients comprised the majority. Among the types of operation that the patients underwent, cardiovascular surgery was the most frequent at $40.8 \%$, followed by nervous system surgery at $40.5 \%$.

In the case of the final patient outcome after surgery, full recovery was $85.7 \%$, no improvement was $4.3 \%$, unverifiable outcomes was $0.7 \%$, discharged patients with no likelihood was $0.4 \%$ and dead patients was $8.9 \%$. normal discharging was the most frequent discharging of patients $88.1 \%$ and 'transfer from other hospitals' was $9.3 \%$, and 265 patients $(36.4 \%)$ were admitted as an outpatient and $462(63.5 \%)$ were hospitalized through the emergency room. For hospitalization route, direct visit $(73.5 \%)$ was higher than request from other medical institutions, and health insurance $(88.6 \%)$ comprised the majority of primary payer of medical fees and medical benefit was $7.8 \%$. The average number of days of hospitalization was $37.9 \pm 72.0$ days.

Table 4. Patient Characteristics

\begin{tabular}{lc}
\hline \multicolumn{1}{c}{ Categories } & $\mathrm{n}(\%)$ or M $\pm \mathrm{SD}$ \\
\hline Age & $56.8 \pm 16.9$ \\
$\mathrm{Sex}$ & $351(48.2)$ \\
Female & \\
Type of disease(ICD-10) & $194(26.7)$ \\
I61(Intracerebral hemorrhage) & $133(18.3)$ \\
I62(Other nontraumatic intracranial hemorrhage) & $71(9.8)$ \\
I63(Cerebral infarction) & $115(15.8)$ \\
I65(Occlusion and stenosis of precerebral arteries, not resulting in & $36(5.0)$ \\
$\quad$ cerebral infarction) & $175(24.0)$ \\
I67(Other cerebrovascular disease) & $4(0.6)$ \\
I69(Sequelae of cerebrovascular disease) & $295(40.5)$ \\
Type of surgery(ICD-9-CM) & $2(0.3)$ \\
I . Operations on the nervous system & $2(0.3)$ \\
III. Operations on the eye & $17(2.3)$ \\
V. Operations on the nose, mouth, and pharynx & $297(40.8)$ \\
VI. Operations on the respiratory system & $14(1.9)$ \\
VII. Operations on the cardiovascular system & $5(0.7)$ \\
IX. Operations on the digestive system & $14(1.9)$ \\
X. Operations on the urinary system & $6(0.8)$ \\
X IV. Operations on the musculoskeletal system & $76(10.4)$ \\
X V. Operations on the integumentary system & \\
X VI. Miscellaneous diagnostic and therapeutic procedures & $159(21.8)$ \\
Residence & $157(21.6)$ \\
Special city & $327(44.9)$ \\
Metropolitan cities & $85(11.7)$ \\
Administrative city areas &
\end{tabular}




\begin{tabular}{lc} 
Administrative county areas & \\
Treatment result & $624(85.7)$ \\
Full(partial) recovery & $31(4.3)$ \\
No improvement & $5(0.7)$ \\
Only diagnosis & $3(0.4)$ \\
Hopeless discharge & $65(8.9)$ \\
Death & \\
Type of discharge & $642(88.1)$ \\
Normal discharge & $13(1.8)$ \\
Discharge against medical advice & $68(9.3)$ \\
Transfer to other hospitals & $5(0.7)$ \\
Run away from hospital & \\
Route of admission & $265(36.4)$ \\
Outpatient department & $462(63.5)$ \\
Emergency room & $1(0.1)$ \\
Etc. & \\
Hospital visit courses & $535(73.5)$ \\
Direct visit & $193(26.5)$ \\
Refer from other hospitals & \\
The primary payer of medical fees & $12(1.7)$ \\
Paid full medical expense & $645(88.6)$ \\
Health insurance & $8(1.1)$ \\
Occupational health and safety insurance & $3(0.4)$ \\
Car insurance & $57(7.8)$ \\
Medical benefit & $3(0.4)$ \\
Etc. & $37.9 \pm 72.0$ \\
The number of days of hospitalization & $39.0 \pm 88.4$ \\
\hline
\end{tabular}

\subsection{Mortality by Patient Characteristics}

The 728 surgical cerebrovascular disease patients were categorized into mortality and non-mortality cases, and their characteristics were examined. Sixty-five patients died including 35 male, and their average age was $58.0 \pm 15.1$. Intracerebral hemorrhage was the cause of death in $44.6 \%$ and subarachnoid hemorrhage in $35.4 \%$, comprising the majority; furthermore, nervous system surgery had been conducted in $60 \%$ of the dead patients and cardiovascular surgery in $18.5 \%$. Patients' residence was evenly distributed throughout the special city, metropolitan cities and administrative city areas, and $92.3 \%$ were hospitalized through the emergency room, $67.7 \%$ of the patients had directly visited. $89.2 \%$ paid for medical fees through health insurance and $9.2 \%$ through medical benefit; and the average number of days of hospitalization was $39.0 \pm 88.4$ days. .

A total of 663 surgical cerebrovascular disease patients were non-mortality cases. Their average age was $56.7 \pm 17.0$ years old, and $51.6 \%$ of them were males. Subarachnoid hemorrhage accounted for $25.8 \%$, other cerebrovascular disease accounted for $25.9 \%$ and cerebral infarction accounted for $16.0 \%$, and $43.0 \%$ of the patients received cardiovascular surgery while $38.6 \%$ of the patients received nervous system surgery. The majority of the patients $(46.3 \%)$ resided in city areas, and the reported hospitalization route was $60.6 \%$ through the emergency room and $74.1 \%$ through direct visiting. Health insurance was the primary payer of medical fees in $88.5 \%$ and medical benefit in $7.7 \%$; and the average number of days of hospitalization was $37.8 \pm 70.2$.

The categories of variables applied to multilevel analysis were reconstituted as follows: type of disease was categorized into I60 (subarachnoid hemorrhage), I61 (intracerebral hemorrhage), I63 (cerebral infarction), I67 (other cerebrovascular disease) and others; types of operation into nervous system surgery, cardiovascular surgery and others; treatment outcomes into full (partial) recovery, no improvement, mortality and others; 
hospitalization route into the emergency room, as outpatient and others; primary payer into health insurance, medical benefit and others.

\section{Table 5. Mortality by Patient Characteristics}

\begin{tabular}{|c|c|c|}
\hline Categories & $\begin{array}{c}\text { Mortality } \\
\mathrm{n}(\%) \text { or } \mathrm{M} \pm \mathrm{SD} \\
\end{array}$ & $\begin{array}{r}\text { Non-mortality } \\
\mathrm{n}(\%) \text { or } \mathrm{M} \pm \mathrm{SD} \\
\end{array}$ \\
\hline Age & $58.0 \pm 15.1$ & $56.7 \pm 17.0$ \\
\hline \multicolumn{3}{|l|}{ Sex } \\
\hline Male & $35(53.9)$ & 342(51.6) \\
\hline Female & $30(46.2)$ & $321(48.4)$ \\
\hline \multicolumn{3}{|l|}{ Type of disease(ICD-10) } \\
\hline I60(Subarachnoid hemorrhage) & $23(35.4)$ & 171(25.8) \\
\hline I61(Intracerebral hemorrhage) & 29(44.6) & 104(15.7) \\
\hline I62(Other nontraumatic intracranial hemorrhage) & $1(1.5)$ & $70(10.6)$ \\
\hline I63(Cerebral infarction) & $9(13.9)$ & 106(16.0) \\
\hline $\begin{array}{l}\text { I65(Occlusion and stenosis of precerebral arteries, not resulting } \\
\text { in cerebral infarction) }\end{array}$ & $0(0)$ & $36(5.4)$ \\
\hline I67(Other cerebrovascular disease) & 3(4.6) & 172(25.9) \\
\hline I69(Sequelae of cerebrovascular disease) & $0(0)$ & $4.0(0.6)$ \\
\hline \multicolumn{3}{|l|}{ Type of surgery(ICD-9-CM) } \\
\hline I . Operations on the nervous system & $39(60.0)$ & 256(38.6) \\
\hline III. Operations on the eye & $0(0)$ & $2(0.3)$ \\
\hline V. Operations on the nose, mouth, and pharynx & $0(0)$ & $2(0.3)$ \\
\hline VI. Operations on the respiratory system & $2(3.1)$ & $15(2.3)$ \\
\hline VII. Operations on the cardiovascular system & $12(18.5)$ & $285(43.0)$ \\
\hline IX. Operations on the digestive system & $3(4.6)$ & $11(1.7)$ \\
\hline $\mathrm{X}$. Operations on the urinary system & 2(3.1) & $3(0.5)$ \\
\hline X IV. Operations on the musculoskeletal system & $0(0)$ & 14.0(2.1) \\
\hline X V. Operations on the integumentary system & $1(1.5)$ & $5(0.8)$ \\
\hline X VI. Miscellaneous diagnostic and therapeutic procedures & $6(9.2)$ & 70(10.6) \\
\hline \multicolumn{3}{|l|}{ Residence } \\
\hline Special city & $20(30.8)$ & 139(21.0) \\
\hline Metropolitan cities & $20(30.8)$ & 137(20.7) \\
\hline Administrative city areas & $20(30.8)$ & 307(46.3) \\
\hline Administrative county areas & $5(7.7)$ & $80(12.1)$ \\
\hline \multicolumn{3}{|l|}{ Treatment result } \\
\hline Full(partial) recovery & $0(0)$ & 642(94.1) \\
\hline No improvement & $0(0)$ & $31(4.7)$ \\
\hline Only diagnosis & $0(0)$ & $5(0.8)$ \\
\hline Hopeless discharge & $0(0)$ & $3(0.5)$ \\
\hline Death & $65(100)$ & $0(0)$ \\
\hline \multicolumn{3}{|l|}{ Route of admission } \\
\hline Outpatient department & $5(7.7)$ & 260(39.2) \\
\hline Emergency room & $60(92.3)$ & $402(60.6)$ \\
\hline Etc. & $0(0)$ & $1(0.2)$ \\
\hline \multicolumn{3}{|l|}{ Hospital visit courses } \\
\hline Direct visit & $44(67.7)$ & 491(74.1) \\
\hline Refer from other hospitals & $21(32.3)$ & 172(25.9) \\
\hline \multicolumn{3}{|l|}{ The primary payer of medical fees } \\
\hline Paid full medical expense & $0(0)$ & $12(1.8)$ \\
\hline Health insurance & $28(89.2)$ & $587(88.5)$ \\
\hline Occupational health and safety insurance & $0(0)$ & $8(1.2)$ \\
\hline Car insurance & $0(0)$ & $3(0.5)$ \\
\hline Medical benefit & $6(9.2)$ & $541(7.7)$ \\
\hline
\end{tabular}


Etc.
$1(1.5)$

\subsection{Length of Hospital Stay by Patient Characteristics}

Surgical cerebrovascular disease patients aged between 20-39 were hospitalized for the longest duration, and patients diagnosed with subarachnoid hemorrhage, intracerebral hemorrhage, cerebral infarction displayed similar lengths of hospitalization; however, the longest was subarachnoid hemorrhage. Patients who received musculoskeletal surgery were hospitalized for the longest duration followed by those who received nervous system surgery, and patients who displayed no improvement showed longer hospital stay than cases where the patients had died. In the case of transfer, the number of days of hospitalization was long; and the length of hospital stay of those hospitalized through the emergency room was longer than those hospitalized as outpatients; and the length of hospital stay of those referred by other medical institution was longer than those who had directly visited; and patients of occupational health and safety insurance or car insurance were hospitalized for 5 times longer than health insurance patients.

Table 6. Length of Hospital Stay by Patient Characteristics (Days)

\begin{tabular}{|c|c|}
\hline Categories & $\mathrm{M} \pm \mathrm{SD}$ \\
\hline \multicolumn{2}{|l|}{ Age } \\
\hline $1-19$ years & $29.2 \pm 65.5$ \\
\hline $20-39$ years & $46.4 \pm 92.1$ \\
\hline $40-59$ years & $41.6 \pm 79.8$ \\
\hline $60-79$ years & $35.0 \pm 63.4$ \\
\hline Over 80 years & $29.8 \pm 38.7$ \\
\hline \multicolumn{2}{|l|}{ Sex } \\
\hline Male & $36.2 \pm 66.0$ \\
\hline Female & $39.6 \pm 77.9$ \\
\hline \multicolumn{2}{|l|}{ Type of disease(ICD-10) } \\
\hline I60(Subarachnoid hemorrhage) & $51.8 \pm 96.2$ \\
\hline I61(Intracerebral hemorrhage) & $48.7 \pm 63.4$ \\
\hline I62(Other nontraumatic intracranial hemorrhage) & $26.0 \pm 60.5$ \\
\hline I63(Cerebral infarction) & $47.8 \pm 80.5$ \\
\hline $\begin{array}{l}\text { I65(Occlusion and stenosis of precerebral arteries, not resulting in cerebral } \\
\text { infarction) }\end{array}$ & $10.8 \pm 12.0$ \\
\hline I67(Other cerebrovascular disease) & $18.8 \pm 40.2$ \\
\hline I69(Sequelae of cerebrovascular disease) & $19.8 \pm 10.4$ \\
\hline \multicolumn{2}{|l|}{ Type of surgery(ICD-9-CM) } \\
\hline I . Operations on the nervous system & $45.9 \pm 80.3$ \\
\hline III. Operations on the eye & $29.0 \pm 7.1$ \\
\hline V. Operations on the nose, mouth, and pharynx & $26.0 \pm 19.8$ \\
\hline VI. Operations on the respiratory system & $42.2 \pm 25.8$ \\
\hline VII. Operations on the cardiovascular system & $34.2 \pm 65.5$ \\
\hline IX. Operations on the digestive system & $16.6 \pm 13.7$ \\
\hline $\mathrm{X}$. Operations on the urinary system & $26.2 \pm 33.4$ \\
\hline X IV. Operations on the musculoskeletal system & $66.9 \pm 144.4$ \\
\hline $\mathrm{X} V$. Operations on the integumentary system & $38.0 \pm 44.1$ \\
\hline X VI. Miscellaneous diagnostic and therapeutic procedures & $20.4 \pm 56.3$ \\
\hline \multicolumn{2}{|l|}{ Residence } \\
\hline Special city & $35.2 \pm 57.9$ \\
\hline
\end{tabular}




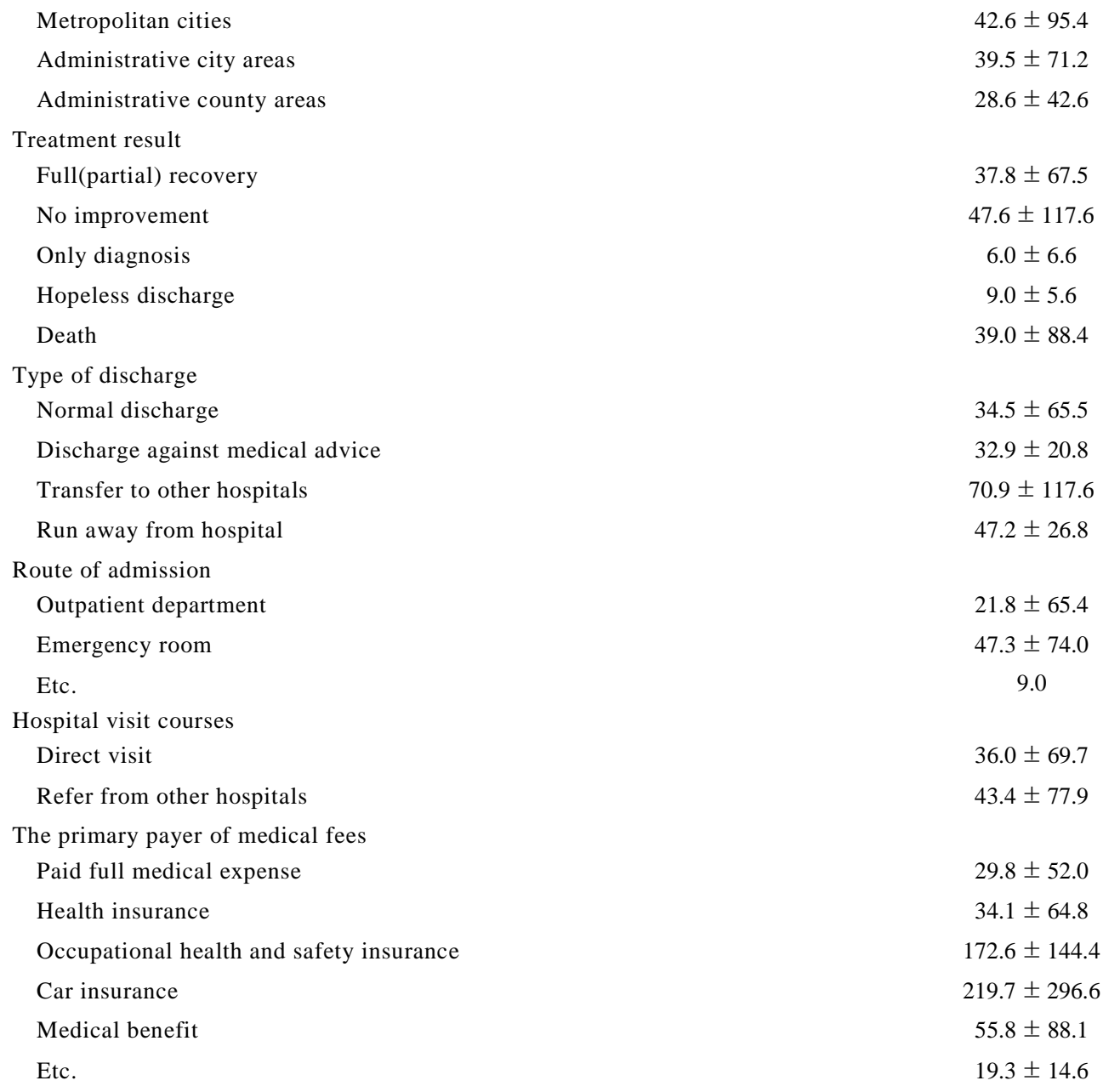

\subsection{Association between Nurse Staffing and Inpatient Mortality}

To determine the influences of nurse staffing on inpatient mortality, models were constructed with variables of hospital standards and patient standards, as surgical cerebrovascular disease patients as subjects. Nurse staffing variable was applied to each model. For mortality of surgical cerebrovascular disease patients, types of their disease and the operation they received displayed a significant relationship, whereas, the indicators explaining mortality and nurse staffing did not have a significant relationship.

In Model 1 that includes number of beds per nurse, subarachnoid hemorrhage displayed 15.92 times higher mortality rate than other disease (other nontraumatic intracranial hemorrhage, occlusion and stenosis of precerebral arteries, not resulting in cerebral infarction, sequel of cerebrovascular disease); Intracerebral hemorrhage displayed 16.96 times higher mortality rate than other disease; and other surgeries displayed 0.32 times lower mortality rate than cardiovascular surgery. However, the relationship between the indicator of nurse staffing, number of beds per nurse and mortality was not significant.

In Model 2 that included number of inpatient days per nurse, subarachnoid hemorrhage displayed 16.10 higher mortality rate than other diseases (other nontraumatic intracranial hemorrhage, occlusion and stenosis of precerebral arteries, not resulting in cerebral infarction, sequel of cerebrovascular disease); Intracerebral hemorrhage displayed 16.10 times higher mortality rate than other diseases; cardiovascular surgery displayed 0.33 times lower mortality rate than other surgeries. However, the relationship between number of inpatient days per nurse and mortality was not significant. 
Table 7. Multilevel Logistic Regression Summary for Mortality

\begin{tabular}{|c|c|c|c|c|c|c|c|c|}
\hline \multirow{3}{*}{ Categories } & \multicolumn{4}{|c|}{ Mortality } & \multicolumn{4}{|c|}{ Non-mortality } \\
\hline & \multirow{2}{*}{$\mathrm{OR}$} & \multicolumn{2}{|c|}{$95 \% \mathrm{CI}$} & \multirow{2}{*}{$p$} & \multirow{2}{*}{$\mathrm{OR}$} & \multicolumn{2}{|c|}{$95 \% \mathrm{CI}$} & \multirow{2}{*}{$p$} \\
\hline & & Upper & Lower & & & Upper & Lower & \\
\hline The number of bed per a nurse & 0.51 & 0.14 & 1.86 & .309 & & & & \\
\hline The number of inpatient per a nurse & & & & & 1.17 & 0.23 & 5.94 & .854 \\
\hline \multicolumn{9}{|l|}{ The number of bed(vs over 500 bed) } \\
\hline $100-499$ bed & 1.00 & 0.12 & 8.74 & .998 & 0.64 & 0.07 & 5.69 & .691 \\
\hline \multicolumn{9}{|l|}{ Type of establish(vs private) } \\
\hline Public & 1.05 & 0.40 & 2.77 & .919 & 0.98 & 0.37 & 2.66 & .975 \\
\hline \multicolumn{9}{|l|}{ Training physician or not(vs not) } \\
\hline Training hospital & 4.82 & 0.30 & 76.92 & .265 & 3.84 & 0.25 & 60.14 & .337 \\
\hline \multicolumn{9}{|l|}{ Location of hospitals (vs etc.) } \\
\hline Special city & 0.92 & 0.30 & 2.88 & .891 & 1.10 & 0.34 & 3.50 & .876 \\
\hline Metropolitan cities & 1.03 & 0.35 & 3.00 & .958 & 1.03 & 0.35 & 3.06 & .960 \\
\hline The number of bed per a physician & 1.26 & 0.93 & 1.70 & .141 & 1.21 & 0.89 & 1.64 & .216 \\
\hline \multicolumn{9}{|l|}{ Sex(vs female) } \\
\hline Male & 1.21 & 0.67 & 2.19 & .528 & 1.22 & 0.67 & 2.20 & .518 \\
\hline Age & 1.00 & 0.98 & 1.02 & .922 & 1.00 & 0.98 & 1.02 & .879 \\
\hline \multicolumn{9}{|l|}{ Type of diseases (vs etc.) } \\
\hline I60(Subarachnoid hemorrhage) & 15.92 & 1.92 & 131.92 & .010 & 15.31 & 1.85 & $\begin{array}{c}126.5 \\
9\end{array}$ & .011 \\
\hline I61(Intracerebral hemorrhage) & 16.96 & 2.13 & 135.25 & .008 & 16.10 & 2.02 & $\begin{array}{c}128.4 \\
0\end{array}$ & .009 \\
\hline I63(Cerebral infarction) & 6.50 & 0.74 & 57.44 & .092 & 6.37 & 0.72 & 56.20 & .096 \\
\hline I67(Other cerebrovascular disease) & 4.11 & 0.36 & 46.59 & .254 & 4.02 & 0.36 & 45.52 & .260 \\
\hline \multicolumn{9}{|l|}{ Type of operations (vs etc.) } \\
\hline I . Operations on the nervous system & 0.97 & 0.42 & 2.27 & .945 & 0.96 & 0.41 & 2.25 & .922 \\
\hline VII. Operations on the cardiovascular system & 0.32 & 0.12 & 0.90 & .031 & 0.33 & 0.12 & 0.93 & .037 \\
\hline \multicolumn{9}{|c|}{ Residence of patients(vs Administrative county areas) } \\
\hline Special city & 1.87 & 0.53 & 6.59 & .331 & 1.85 & 0.53 & 6.55 & .337 \\
\hline Metropolitan cities & 1.65 & 0.47 & 5.81 & .436 & 1.73 & 0.49 & 6.13 & .397 \\
\hline Administrative city areas & 0.82 & 0.27 & 2.49 & .726 & 0.84 & 0.28 & 2.54 & .757 \\
\hline \multicolumn{9}{|l|}{ Route of admission(vs emergency room) } \\
\hline Outpatient department and etc. & 0.34 & 0.11 & 1.09 & .070 & 0.36 & 0.11 & 1.13 & .080 \\
\hline \multicolumn{9}{|l|}{ Hospital visit courses(vs Refer from other hospitals) } \\
\hline Direct visit & 0.90 & 0.45 & 1.78 & .756 & 0.88 & 0.44 & 1.75 & .705 \\
\hline \multicolumn{9}{|c|}{ The primary payer of medical fees(vs Health insurance) } \\
\hline Etc. & 0.19 & 0.02 & 1.53 & .118 & 0.18 & 0.02 & 1.50 & .113 \\
\hline Medical benefit & 0.96 & 0.36 & 2.56 & .935 & 0.94 & 0.35 & 2.51 & .905 \\
\hline
\end{tabular}

Note: $\mathrm{OR}=$ Odds ratio; $\mathrm{CI}=$ Confidence interval

\subsection{Association between Nurse Staffing and Length of Hospital Stay}

Study models, containing all hospital and patient standards variables, were established in order to determine the relationship between nurse staffing and length of hospitalization in surgical cerebrovascular disease patients. Surgical cerebrovascular disease patients displayed a statistically significant relationship between type of disease, type of surgery, admission route and primary payer, and among indicators associated with nurse staffing, number of beds per nurse, but not the number of inpatient days, displayed significant relationship.

For patient standards variable in Model 1, which includes the Medical Service Act estimated number of beds per nurse, subarachnoid hemorrhage, intracerebral hemorrhage, cerebral infarction, other cerebrovascular diseases displayed longer hospital stay than 
disease classified as others (other nontraumatic intracranial hemorrhage, occlusion and stenosis of precerebral arteries, not resulting in cerebral infarction, sequel of cerebrovascular disease); and those who had nervous system surgery, those who had cardiovascular surgery were hospitalized for longer than those who received other types of surgeries. Furthermore, those hospitalized as outpatients or through other routes were in hospital for a shorter period than those hospitalized through the emergency room; those who paid through health insurance were hospitalized for a shorter time period than those who paid through other forms of payment (one's own money, occupational health and safety insurance, car insurance, others) or medical benefit. Among the indicators associated with nurse staffing, increase in the number of beds per nurse caused lengthened hospital stays.

For patient standards variable in Model 2, which includes the Medical Service Act estimated number of inpatient days per nurse, subarachnoid hemorrhage, intracerebral hemorrhage, cerebral infarction, other cerebrovascular diseases as others (other nontraumatic intracranial hemorrhage, occlusion and stenosis of precerebral arteries, not resulting in cerebral infarction, sequel of cerebrovascular disease); and those who nervous system surgery, those who received cardiovascular surgery were hospitalized for longer than those who received other surgeries. Those hospitalized as outpatients or through other routes were in hospital for shorter time period than those hospitalized through the emergency room; those who paid through health insurance were hospitalized for a shorter time period than those who paid through other payment methods or medical benefit. However, the number of inpatient days per nurse did not have a significant relationship with the number of days of hospitalization.

Table 8. Multilevel Linear Regression Summary for Length of Hospital Stay

\begin{tabular}{|c|c|c|c|c|c|c|c|c|}
\hline \multirow[b]{2}{*}{ Categories } & \multicolumn{4}{|c|}{ Mortality } & \multicolumn{4}{|c|}{ Non-mortality } \\
\hline & Estimate & SE & $\mathrm{t}$ & $p$ & Estimate & SE & $\mathrm{t}$ & $p$ \\
\hline The number of bed per a nurse & 0.587 & 0.276 & 2.13 & .034 & & & & \\
\hline The number of inpatient per a nurse & & & & & 0.408 & 0.360 & 1.13 & .257 \\
\hline \multicolumn{9}{|l|}{ The number of bed(vs over 500 bed) } \\
\hline $100-499$ bed & -0.530 & 0.468 & -1.13 & .258 & -0.358 & 0.480 & -0.75 & .456 \\
\hline \multicolumn{9}{|l|}{ Type of establish(vs private) } \\
\hline Public & -0.104 & 0.185 & -0.56 & .573 & -0.111 & 0.195 & -0.57 & .568 \\
\hline \multicolumn{9}{|l|}{ Training physician or not(vs not) } \\
\hline Training hospital & -0.342 & 0.621 & -0.55 & .582 & -0.113 & 0.635 & -0.18 & .859 \\
\hline \multicolumn{9}{|l|}{ Location of hospitals (vs etc.) } \\
\hline Special city & 0.089 & 0.203 & 0.44 & .660 & 0.035 & 0.211 & 0.17 & .869 \\
\hline Metropolitan cities & 0.034 & 0.196 & 0.17 & .862 & 0.057 & 0.203 & 0.28 & .779 \\
\hline The number of bed per a physician & -0.038 & 0.064 & -0.59 & .557 & -0.018 & 0.066 & -0.27 & .787 \\
\hline \multicolumn{9}{|l|}{ Sex(vs female) } \\
\hline Male & -0.096 & 0.079 & -1.21 & .227 & -0.097 & 0.079 & -1.22 & .222 \\
\hline Age & -0.003 & 0.003 & -1.23 & .220 & -0.003 & 0.003 & -1.17 & .242 \\
\hline \multicolumn{9}{|l|}{ Type of diseases (vs etc.) } \\
\hline I60(Subarachnoid hemorrhage) & 0.379 & 0.142 & 2.67 & .008 & 0.384 & 0.142 & 2.70 & .007 \\
\hline I61(Intracerebral hemorrhage) & 0.550 & 0.146 & 3.78 & .001 & 0.558 & 0.146 & 3.82 & .001 \\
\hline I63(Cerebral infarction) & 0.875 & 0.148 & 5.93 & $<.0001$ & 0.881 & 0.148 & 5.96 & $<.0001$ \\
\hline I67(Other cerebrovascular disease) & 0.258 & 0.145 & 1.78 & .075 & 0.258 & 0.145 & 1.78 & .076 \\
\hline \multicolumn{9}{|l|}{ Type of operations (vs etc.) } \\
\hline I . Operations on the nervous system & 0.698 & 0.129 & 5.39 & $<.0001$ & 0.705 & 0.130 & 5.43 & $<.0001$ \\
\hline VII. Operations on the cardiovascular system & 0.741 & 0.131 & 5.64 & $<.0001$ & 0.742 & 0.132 & 5.61 & $<.0001$ \\
\hline \multicolumn{9}{|c|}{ Residence of patients(vs Administrative county areas) } \\
\hline Special city & -0.127 & 0.152 & -0.84 & .403 & -0.130 & 0.152 & -0.86 & .393 \\
\hline
\end{tabular}




$\begin{array}{lcccccccc}\text { Metropolitan cities } & -0.126 & 0.149 & -0.84 & .399 & -0.130 & 0.149 & -0.87 & .384 \\ \quad \text { Administrative city areas } & -0.038 & 0.126 & -0.30 & .763 & -0.045 & 0.126 & -0.35 & .724 \\ \begin{array}{l}\text { Route of admission(vs emergency room) } \\ \text { Outpatient department and etc. }\end{array} & & & & & & & & \\ \begin{array}{l}\text { Hospital visit courses(vs Refer from other hospitals) } \\ \quad \text { Direct visit }\end{array} & -0.617 & 0.111 & -5.59 & <.0001 & -0.620 & 0.111 & -5.60 & <.0001 \\ \begin{array}{l}\text { The primary payer of medical fees(vs Health } \\ \text { insurance) }\end{array} & 0.023 & 0.099 & 0.23 & .820 & 0.024 & 0.100 & 0.24 & .814 \\ \quad \text { Etc. } & & & & & & & & \\ \quad \text { Medical benefit } & 0.539 & 0.216 & 2.49 & .013 & 0.541 & 0.217 & 2.50 & .013 \\ \end{array}$

Note: $\mathrm{SE}=$ Stand error

\section{Discussion}

This study is a descriptive correlation study that determines the relationship between nurse staffing and patient outcomes (mortality, length of hospital stay) by utilizing multilevel analysis that considers characteristics of hospital standards and patient standards.

The conclusions of the study's mortality index indicated that there are no significant relationships between mortality and both the number of beds per nurse and the number of inpatient days per nurse. Such conclusion contradicts results from previous studies [2022] on nurse staffing and inpatient mortality among patient outcomes, which displayed that increased nurse staffing leads to decreased mortalities of patients within the hospital. This may have resulted from the limitations of data in this study and consequential lack of sufficient estimation of patient outcomes; patients' characteristics, the structure and the process of treatment provision, and other various variables among which patients' characteristics have the largest influence on mortalities [23-24] and the effect of patient treatment and hospital structure can be measured through effective hazard estimation of patients' characteristics. Halm et al [25] explains that the failure to reflect the patient severity between hospitals accounts for the lack of significant relationship between nurse staffing and mortality of patients in studies with general surgery, orthopedics, and vascular surgery patients as subjects.

Moreover, such results can also be explained by the short average clinical career of domestic nurses. Cho et al [17] in a study on the relationship between ICU nurse staffing and patient mortalities, showed that the domestic nurses' average clinical career was as short as 5.5 years, as compared to 9 years in Taiwan, Ontario and Pennsylvania[26-27]. Short nursing career may have prevented nurse staffing from sufficiently influencing patients' mortalities. Thus, further studies should reflect qualitative factors such as nurses' clinical career, level of education, and shifts etc.

However, in a study conducted by Pronovost et al [11], there was no disparity when 1 ICU nurse was responsible for $<2$ patients who received abdominal aortic aneurysm surgery or $>2$ patients; Needleman et al [13] included both internal medicine and surgical patients as subjects in their study and showed no significant influences on mortality by nurse staffing in both cases, corroborating the conclusions of this study.

Lang et al [20] concluded that despite the several reports on the relationship between mortality and nurse staffing, the results are not consistent. Also Needlman et al [13] reported the absence of any relationship between mortality and nurse staffing tin internal medicine patients, but in surgical patients with side effects, mortality is dependent on nurse staffing. Thus, objective and continuous study on nurse staffing and patient outcomes that measure severity of patients per patient group are required.

The second patient outcome indicator of this study, i.e., the length of hospital stay, showed that as the number of beds per nurse increased, length of hospital stay increased significantly for surgical cerebrovascular disease patients. This coincides with the 
reported results of Amaravadi [2] where the median number of hospitalization days is significantly higher when the ratio of ICU night shift nurses to patients who received thoracic esophagectomy is over $1: 2$, as compared to when the ratio is below $1: 2$; in addition, Park [5] studied direct nursing activity per sliding scale of nursing management costs and reported that the length of hospital stay index is lower in grade 6 on, similar to the results of grade 2 and 3 that are excellent. On the other hand, Needleman et al [3] demonstrated that the length of hospital stay was significantly shorter for internal medicine patients but surgical patients displayed no difference in the length of hospital stay, indicating a partial influence on the number of hospitalization days .

Multilevel analysis for the influences on the number of hospitalization days using the patient standards and hospital standards variables of surgical cerebrovascular disease patients displayed that one of the indicators associated with nurse staffing, i.e., the number of beds per nurse was of significant explanatory power. This corroborates preceding research that emphasized the need for experienced nurses in wards with cerebral apoplexy patients than general ward, to reduce mortality and disabilities after cerebral apoplexy; thus, arbitration provided by nurses is highly important for cerebrovascular disease patients [28]. The American Nurses Association explains that low nurse staffing can increase the probability of mistakes as nurses are highly occupied, leading to poor quality control; whereas, adequate nurse staffing means higher allocation of time per patient in every aspect of patient treatment; furthermore, preceding studies have indicated that low nurse staffing results in decreased patient monitoring, delayed or omitted patient treatment, poor recording and increased injection accidents [29]. However, Shamian et al [30] reported that the shorter length of hospital stay at nursing departments with higher nurse staffing was due to the increased demand for short-term nursing such as discharge training and future treatment related to rehabilitation, and the hospital thus introduces more nurses to their staff.

Number of hospitalization days is inter-related with patients' burden of medical fees and hospital profit. Reasonable management of length of hospital stay benefits medical consumers, suppliers and the nation. In other words, shortened length of hospital stay leads to increase in bed circulation rate, enhances treatment of new patients, and improves the hospitals' profit. Also, it benefits the patients as they are discharged on rapid health improvement after surgery and acute treatment, thus saving on medical fees. Shortening of length of hospital stay is an important factor that brings profit to the hospitals and reduces patients' medical fees [31]. Thus, maintaining adequate level of nursing staff is expected to have positive effects on length of hospital stay management.

\section{Conclusion}

This study is a multilevel analysis of large-scale national data from general hospitals to determine the influences of nurse staffing on patient outcomes (mortality and length of hospital stay). Using general hospitals in the 2008 Korea National Patients Survey, 286 hospitals that treat ischemic heart diseases, cerebrovascular disease inpatients were examined and the patients were categorized into internal medicine and surgical patients, in order to objectively determine the influences of nurse staffing on patient outcomes.

Multilevel analysis for factors that influence the length of hospital stay of surgical cerebrovascular disease patients showed that increases in Medical Service Act estimated number of beds per nurse led to significant lengthening of the patients' length of hospital stay. This study results indicated that decreasing nurse staffing led to increase in the number of days of hospitalization for surgical cerebrovascular disease patients. Our results could serve as evidence for the need to maintain adequate nurse staffing in the future. Furthermore, we included multilevel analysis on the influences of nurse staffing on patient outcomes by estimated hospital standards and patient standards, and suggest a 
methodology for study on nurse staffing and patient outcomes using future national data set.

In this study, the relationship between nurse staffing and mortality was not significant. Thus, further study including important variables such as procedural factors including nursing arbitration, an important variable influencing mortality, in addition to the variables already examined in this study is needed. Moreover, supplementary studies that examine other indicators apart from mortality and length of hospital stay through domestic large-scale national data are recommended.

Further development of various indicators associated with nurse staffing as well as research on level of nurse staffing that reflects nurses characteristics in the field including shifts, nursing career, level of nursing education and communication between the medical team is required.

\section{References}

[1] L. H. Aiken, H. L. Smith and E. T. Lake, 'Lower Medicare Mortality among a Set of Hospitals Known for Good Nursing Care', Medical Care vol.32, no.8, (1994), pp.771-787.

[2] R. K. Amaravadi, J. B. Dimick, P. J. Pronovost and P. A. Lipsett, 'ICU nurse to patient ratio is associated with complications and resource use after esophagectomy', Intensive Care Medicine, nol.26, no.12, (2000), pp.1857-1862.

[3] J. Needleman, P. Buerhaus, S. Mattke, M. Stewart and K. Zelevinsky, 'Nurse-staffing levels and the quality of care in hospitals', New England Journal of Medicine, vol.326, no.22, (2002), pp.1715-1722.

[4] S. H. Cho, S. Ketefian, V. H. Barkauskas and D. G. Smith, 'The effects of nurse staffing on adverse events, morbidity, mortality, and medical costs', Nursing Research, (2003), vol.52, no.2, pp.71-79.

[5] S. H. Park, 'Analysis of Direct Nursing Activity and Patient Outcomes Related to Graded Fee of Nursing Management for Inpatient', Journal of Korean Academy of Nursing, (2003), vol.33, no.1, pp.122-129.

[6] S. H. Cho and S. C. Yun, "Bed-to-nurse ratios, provision of basic nursing care, and in-hospital and 30day mortality among acute stroke patients admitted to an intensive care unit: cross-sectional analysis of survey and administrative data", International Journal of Nursing Studies, (2009), vol.46, no.8, pp.10921101.

[7] National Quality Forum, "National Voluntary Consensus Standards for Nursing-Sensitive Care: An Initial Performance Measure Set", A consensus report, National Quality Forum, Washington, DC, (2004).

[8] S. P. Clarke, "Registered nurse staffing and patient outcomes in acute care: looking back, pushing forward", Medical Care, vol.45, no.12, (2007), pp.1126-1128.

[9] R. L. Kane, T. A. Shamliyan, C. Mueller, S. Duval, and T. J. Wilt, "The association of registered nurse staffing levels and patient outcomes: systematic review and meta-analysis", Medical Care, vol.45, no.12, (2007), pp.1195-1204.

[10] S. D. Person, J. J. Allison, C. I. Kiefe, M. T. Weaver, O. D.Williams, R. M. Centor and N. W. Weissman, 'Nurse staffing and mortality for Medicare patients with acute myocardial infarction', Medical Care, vol.42, no.1, (2004), pp.4-12.

[11] P. Pronovost, D. Dang, T. Dorman, P. Lipsett, E. Garrett and M. Jenckes, "Intensive care unit nurse staffing and the risk for complications after abdominal aortic surgery", Effective Clinical Practice, vol.4, no.5, (2001), pp.223-225.

[12] M. Halm, M. Peterson, M. Kandels, J. Sabo, M. Blalock, R. Braden, A. Gryczman, K. Krisko-Hagel, D. Larson, D. Lemay, B. Sisler, L. Strom and D. Topham, 'Hospital nurse staffing and patient mortality, emotional exhaustion, and job dissatisfaction', Clinical Nurse Specialist, vol.19, no.5, (2005), pp.241251.

[13] L. Diya, K. Van den Heede, W. Sermeus and E. Lesaffre, 'The use of "lives saved" measures in nurse staffing and patient safety research: statistical considerations', Nursing Research, vol.60, no.2, (2011), pp.100-106.

[14] Y. M. Kim, 'Nurse Staffing Level Relating Factors of the General Nursing Units, ICU, ER and OR in Acute General Hospitals', The Journal of Korean Nursing Administration Academic Society, vol.14, no.4, (2008), pp.404-412.

[15] S. H. Cho, J. H. Hwang, Y. M. Kim, J. S. Kim, "Variations in Nurse Staffing in Adult and Neonatal Intensive Care Units", The Journal of Korean Academy of Nursing, vol.36, no.5, (2006), pp.691-700.

[16] B. H. Park, K. J. June, Y. M. Kim, 'A Survey on the Nurse Staffing Level and Patient Outcome', The Journal of Korean Nursing Administration Academic Society, vol.9, no.4, (2003), pp.559-569.

[17] S. H. Cho, K. J. June, Y. M. Kim, B. H. Park, 'Changes in Hospital Nurse Staffing after Implementing Differentiated Inpatient Nursing Fees by Staffing Grades', The Journal of Korean Nursing Administration Academic Society, (2008), Vol.14, No.2, pp.167-175. 
[18] S. H. Park, 'Analysis of nursing outcome and direct nursing care activity related to nurse-to-patient ratio', Korea University, Doctoral thesis, (2002).

[19] S. R. Do, 2008 Korea National Patients Survey, The Korea Institute for Health and Social Affairs, Seoul (2009).

[20] T. A. Lang, M. Hodge, V. Olson, P.S. Romano and R.L. Kravitz, 'Nurse patient ratios: a systematic review on the effects of nurse staffing on patient, nurse employee, and hospital outcomes', Journal of Nursing Administration, (2004), vol.34, no.7-8, pp.326-337.

[21] B. A. Mark, D. W. Harless, M. McCue and Y. Xu, 'A longitudinal examination of hospital registered nurse staffing and quality of care', Health Service Research, (2004), vol.39, no.2, pp.279-300.

[22] L. Aiken, S. Clarke , D. Sloane and for the International Hospital Outcomes Research Consortium, ' Hospital nurse staffing and patient mortality, nurse burnout, and job dissatisfaction', The Journal of Amerian Medical Association, (2002), vol.288, no.16, pp.1987-1993.

[23] J. H. Silber, P. R. Rosenbaum and R. N. Ross, 'Comparing the contributions of groups of predictors: which outcomes vary with hospital rather than with patient characteristics?', Journal of the American Statistical Association, (1995), vol.90, no.429, pp.7-18.

[24] J. H. Silber and P. R. Rosenbaum, 'A spurious correlation between hospital mortality and complication rates', Medical Care (1997), vol.30, no.10, pp. OS77-OS92.

[25] M. Halm, M. Peterson, M. Kandels, J. Sabo, M. Blalock, R. Braden, A. Gryczman, K. Krisko-Hagel, D. Larson, D. Lemay, B. Sisler, L. Strom and D. Tophamx, 'Hospital nurse staffing and patient mortality, emotional exhaustion, and job dissatisfaction', Clinical Nurse Specialist (2005), Vol.19, No.5, pp.241251.

[26] L. H. Aiken, S. P. Clarke, R. B. Cheung, D. M. Sloan, J. H. Silber, 'Educational levels of hospital nurses and surgical patient mortality', The Journal of Amerian Medical Association (2003), Vol.290, No.12, pp.1617-1623.

[27] A. Tourangeau, P.Giovannetti, J. Tu and M. Wood, 'Nursingrelated determinants of 30-day mortality for hospitalized patients', The Canadian Journal of Nursing Research, vol.33, no.4, (2002), pp.71-88.

[28] C. R. Burton, A. Fisher and T.L. Green, 'The organisational context of nursing care in stroke units: a case study approach', International Journal of Nursing Studies, vol.46, no.1, (2009), pp.85-94.

[29] American Nurses Association, Implementing Nursing's Report Card: A Study of RN Staffing, Length of Stay and Patient Outcomes. Washington, DC: American Nurses Publishing, (1997).

[30] J. Shamian, B. Hagen, T. W. Hu and T. E. Fogarty, 'The relationship between length of stay and required nursing care hours', Journal of Nursing Administration, (1994), vol. 24, no.7-8, pp.52-58.

[31] Y. H. Kim, J. W. Moon, K. H. Kim, 'The Determinant Factors and Medical Charges Pattern by Length of Stay in Hospital', Korean journal of hospital management, (2010), vol.15, no.2, pp.15-26.

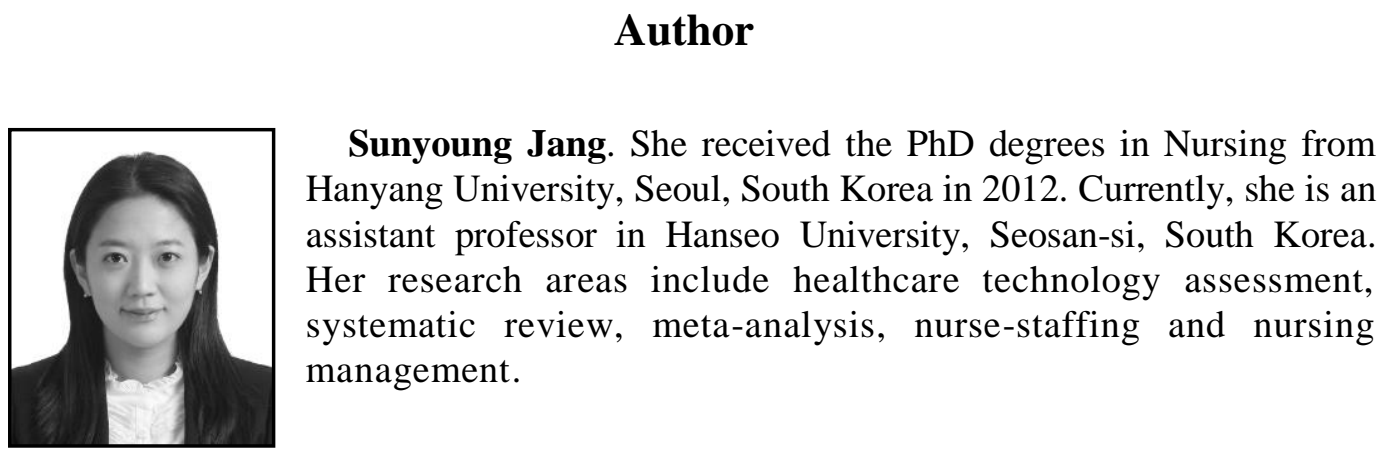


International Journal of Bio-Science and Bio-Technology

Vol.8, No.2 (2016) 